\title{
The effects of a full-aircraft aerodynamic model on the design of a tailored composite wing
}

\author{
Mario Natella ${ }^{1} \cdot$ Roeland De Breuker $^{1}$
}

Received: 14 June 2018 / Revised: 3 January 2019 / Accepted: 28 January 2019 / Published online: 28 February 2019

(c) The Author(s) 2019

\begin{abstract}
This paper discusses the effects of the aerodynamic model on the design of a composite wing via aeroelastic tailoring. The classic framework for analysis and optimization of composite wings developed at Delft University of Technology adopts panel method aerodynamics to calculate static and dynamic loads. The current work expands the aerodynamic model by including fuselage and horizontal tail. The non-linear trim condition is thus calculated taking into account both moment and force equilibrium. The effect the fuselage and horizontal tail have on the load distribution, and relative position between the aerodynamic center and the center of gravity translate into different tailored designs for the composite wings. This study provides insights regarding the use of a full-aircraft aerodynamic model for aeroelastic tailoring optimization.
\end{abstract}

Keywords Aeroelastic tailoring $\cdot$ Composite wing $\cdot$ Structural design

\section{Introduction}

The promise of aeroelastic tailoring was to develop a design framework that could enhance aircraft structural performance at large. The idea sees great recognition in the research community in the 1980 s with important works by Shirk et al. [1] and Weisshaar et al. [2], and recent developments by Stanford et al. [3], and Stodiek et al. [4]. State-ofthe-art aeroelastic tailoring practices have developed frameworks to solve the problem at a wing level. The results in terms of weight saving, load alleviation and/or range optimization are very promising, thus justifying the further development in this field.

The early studies $[1,2]$ explored the potential of aeroelastic tailoring using a single-fiber angle, thus describing most of the fundamental phenomena observed in composite wings and how to prevent them by inducing a beneficial bend-twist coupling.

In the late 1990s, aeroelastic tailoring research started to focus on laminates with different fiber angles through the thickness, an approach that increased the level of complexity of the models to describe more realistic composite

Mario Natella

M.Natella@tudelft.nl

1 ASCM, Delft University of Technology, Kluyverweg 1, 2629 HS Delft, The Netherlands structures. There are three main approaches to this problem found in literature, namely using (1) laminates with a fixed thickness, but varying fiber angles, (2) laminates with a fixed set of fiber angles, and varying thickness and (3) laminates with both varying fiber angles and varying thickness.

The first approach, where the thickness is kept constant, has been solved with evolutionary algorithms [5-7] or fiber steering $[3,4,8]$ to maximize flutter speed. Both approaches already show significant improvements in overall aeroelastic performance due to varying stiffness along the span of the wing compared to the classic approach with straight fibers.

The second approach, with a fixed set of discrete ply angles, provides a solution to comply with certification requirements, reduce the number of design variables [9-11] and improve aeroelastic performance under different set of constraints including buckling, strains and aileron effectiveness.

The aeroelastic framework developed by De Breuker et al. [12] focuses on the third approach where both fiber angles and thickness are modeled as design variables to explore the full potential of aeroelastic tailoring and simplify the formulation of the optimization problem. In contrast to working with fiber angles directly, the formulation where both thickness and fiber angles are varying can be described in the continuous domain using lamination parameters thus making this problem fit for gradient-based solvers and optimization frameworks. The first description 
in lamination parameters was introduced by Kameyama et al. [13]. The approach was first proved for a flat composite panel, where the set of lamination parameters and thickness was calculated for maximum flutter speed. The work of Jin et al. [14], and Dillinger et al. [15] has scaled this approach to solve a similar problem for the whole wing, modeled as a multitude of chordwise and spanwise panels for a more detailed description of a composite wingbox and its structural elements.

In view of a more complete analysis, it is important to understand the classic aeroelastic phenomena in the context of the whole aircraft, in particular with respect to the interaction of the wing with fuselage and tails. The first problem encompasses the solution to the flow around the full-aircraft configuration, in particular in the area near the wing-body junction. A very extensive explanation of the fuselage aerodynamics, including the effects of the wing-body interaction and its importance, is found in the work of Singh et al. [16]. The main contributions of the fuselage aerodynamics are a large pitching moment and a destabilizing neutral point shift upstream. A minor contribution is the increase of effective angle of attack at the root of the wing. The lift generated by the fuselage itself is negligible. Further details regarding the interaction phenomena in a full-aircraft configuration are found in the work of Rusak et al. [17]. Another important point to discuss is the vortex shedding of the body. According to Rusak et al. [17], although there is no comprehensive model to describe the phenomenon, experimental evidence shows how the effect of the body wake on the longitudinal aerodynamic coefficient is of orders of magnitude smaller, thus negligible.

To summarize, the advancement in low- and mediumfidelity physical models for the description of the aeroelastic framework has made new solutions available at the preliminary design level. Already in the early phases of the design process, it is possible to develop a framework for analysis and optimization which is capable to select an improved design candidate for the more detailed phases ahead. Certification aspects and manufacturability constraints are also important parts of the problem that can be incorporated at this level. The benefit of the low-fidelity approach is in the capability of exploring a wide range of the design space at a relative small computational effort or cost. A preliminary study of this size would be almost unfeasible with any 2D or 3D finite element description.

In that spirit, the aim of this paper is to build on the stateof-the-art aeroelastic framework developed at Delft University of Technology by De Breuker et al. [12] and explore the effects of a full-aircraft aerodynamic model on a structurally optimized design via aeroelastic tailoring including material failure, buckling and aileron effectiveness constraints. After an introduction to the aeroelastic framework in Sect. 2, we will discuss the fuselage aerodynamic model (Sect. 3), the full-aircraft aerodynamics (Sect. 5) and aeroelastic solution (Sect. 6). The optimization framework and results are discussed in Sect. 7, while conclusions and recommendation are in Sect. 8.

\section{Aeroelastic framework}

This works builds upon the aeroelastic framework (PROTEUS) developed at Delft University of Technology initiated by De Breuker et al. [12]. The framework serves as an analysis and optimization tool for preliminary design of composite wings. The flow is described by means of a continuous-time state-space model, as extensively described in the work of Werter et al. [18]. The three-dimensional composite wing structure is idealized by a Timoshenko beam by means of a cross-sectional modeler.

The function of the cross-sectional modeler is twofold. First, it is used to calculate the equivalent one-dimensional beam properties starting from the three-dimensional wing model. This is done by means of a thin-walled cross-sectional modeler that was developed based on the work by Willaert et al. [19] and Ferede et al. [20]. The cross-section is discretized in linear Hermitian beam elements having constant properties and can be any arbitrary open or closed, thin-walled, composite cross-section. Using a variational asymptotic approach, the Timoshenko cross-sectional stiffness and mass properties can be determined. The second function of the cross-sectional modeler is the strain recovery. This process converts the one-dimensional beam strains into surface strains. These surface strains include both the Euler-Bernoulli strains and the second-order free warping solution.

The strain field is then used in the assessment of the material failure by means of the failure envelope derived by Ijsselmuiden et al. [21]. The main challenge during the optimization is that the classic composite strength failure criteria cannot be used since the stacking sequence of the laminates is unknown. The failure enveloped is a Tsai-Wubased criterion that ensures no failure regardless of the ply angle. The derivation of the failure envelope criterion as a function of the principal strains is found in Khani et al. [22].

The surface strains are also used to calculate the buckling solution and the buckling index. In a conventional wingbox structure, the buckling panels are defined as the area between two stringers and two ribs. Structural stability is thus assessed in a local sense. Stringers and ribs are assumed to be stiff enough to prevent global buckling. The analytical model is based on the work of Dillinger et al. [23], where the individual panels are assumed to be flat and the bending displacement solution is approximated with 
two-dimensional shape functions obtained from Lobatto (bubble) polynomials.

The aerodynamic and structural models are closely coupled into a unified framework for analysis and optimization. More details regarding the framework and its functionalities are found in the work of Werter et al. [24, 25]. The analytical formulation of the buckling and aileron effectiveness constraint is extensively discussed in Dillinger et al. [15]. The main additions to the state-of-art aeroelastic framework, namely the fuselage aerodynamics (Sect. 3) and the fullaircraft trim solution (Sect. 6), are presented in this paper.

\section{Fuselage aerodynamics}

The aerodynamics of the fuselage is described by means of a source panel distribution. The work by Hess et al. [26] and Katz and Plotkin [27] discusses the two main options for body aerodynamics, namely (1) a source distribution along the center line and (2) a source distribution on the fuselage wetted surface. The first model is more appropriate for bodies of revolution, while for more complex body shapes the second one is preferred. For the purposes of this work, a source distribution on the wetted surface has been adopted.

The fuselage aerodynamics is described by potential flow theory. Flow separation, turbulence and the wake shed by the body are not included in this work. Furthermore, the source panel distribution does not allow for wake modeling. The work by Rusak et al. [17] discusses the challenges of modeling such a physical phenomena should this be of interest to the reader.

The different theories behind the source distribution is given in the work by Katz and Plotkin [27]. A distribution of constant strength source panels has been chosen to describe the aerodynamics of the fuselage. The method is based on the Laplacian of the disturbance velocity potential $\Phi$,

$\nabla^{2} \Phi=0$,

with flow tangency condition,

$\nabla \Phi \cdot \mathbf{n}=\mathbf{0}$,

and the far-field condition as

$\lim _{r \rightarrow \infty} \nabla \Phi=0$

where $r$ refers to the distance from the body. The velocity potential at an arbitrary point $\mathbf{P}$ due to a constant strength quadrilateral source $\sigma$ is:

$\Phi(\mathbf{P})=\frac{\sigma}{4 \pi} \int_{s} \frac{\mathrm{d} S}{\sqrt{\left(x-x_{0}\right)^{2}+\left(y-y_{0}\right)^{2}+z^{2}}}$,

while the local velocity can be derived from the potential $\Phi$ as $\mathbf{u}=\frac{\partial \Phi}{\partial \mathbf{P}}$

By solving Eq. 4, and differentiating with respect to $\mathbf{P}$, one can obtain the expressions for the local velocity components $(u, v$ and $w)$ in each segments, as derived in Katz and Plotkin [27].

Force calculation Hereby the system of equations that describes the flow around the body is given. Neumann boundary conditions are adopted, with the far-field condition being satisfied by definition of the quadrilateral constant strength source panels, and the flow tangency condition enforced. For a single panel, the flow is described by the following equation:

$\mathbf{v} \cdot \mathbf{n}+\mathbf{v}_{\infty} \cdot \mathbf{n}=0$

where $\mathbf{v}^{T}=\left\{v_{x}, v_{y}, v_{z}\right\}$ is the velocity at the collocation point, and $\mathbf{v}_{\infty}$ is the far-field velocity vector in the body reference frame; thus,

$\mathbf{v}_{\infty}=\left[\begin{array}{lll}V_{\infty} \cos \alpha, & 0, V_{\infty} \sin \alpha\end{array}\right]$.

Applying the same logic to the rest of the body panels, the following system of equations is written as

$\mathbf{A} \sigma=-\mathbf{B}$,

with $\mathbf{A}$ being the aerodynamic influence coefficient matrix, $\sigma$ the source strength, and $\mathbf{B}$ the vector with its $i$ th element being the product $\left(\mathbf{v}_{i} \cdot \mathbf{n}_{i}\right)$ for the $i$ th panel.

Once the source strength distribution is known, the panel velocities can be calculated. The equations are written for the velocity components of one panel. Assembling the equations for all the panels leads to a system of equations in matrix format for each velocity component; thus,

$\left\{\begin{array}{r}\mathbf{v}_{x}=\mathbf{A}_{x} \sigma \\ \mathbf{v}_{y}=\mathbf{A}_{y} \sigma \\ \mathbf{v}_{z}=\mathbf{A}_{z} \sigma,\end{array}\right.$

where $\mathbf{v}_{x}, \mathbf{v}_{y}$ and $\mathbf{v}_{z}$ are vectors containing, respectively, the $x, y$ and $z$ components of the velocity in each panel. The pressure distribution is then derived as a function of the panel velocities. The pressure coefficient is generally expressed as

$C_{\mathrm{p}}=1-\frac{V^{2}}{V_{\infty}^{2}}$.

For a single panel $k$, let $\mathbf{R}_{k}$ be the transformation matrix from the body-frame to the local panel coordinates, the local velocity vector can be written as

$\mathbf{v}_{k}=\mathbf{R}_{k}\left(\mathbf{v}_{\infty}+\mathbf{v}_{k}\right)$,

thus, 


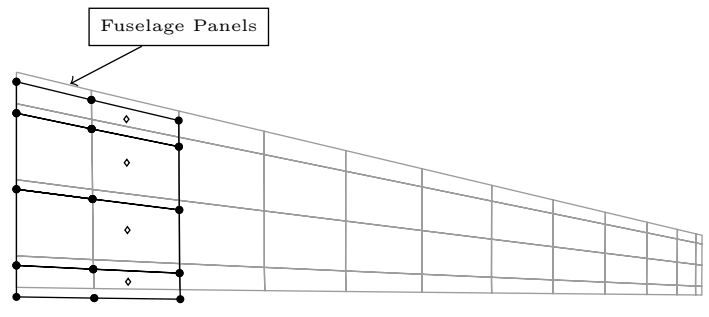

Fig. 1 Detail of wing-fuselage intersection. Diamond collocation points, circle corner points

$C_{\mathrm{p}}=1-\frac{\left\|\mathbf{v}_{k}\right\|^{2}}{V_{\infty}}$.

The force vector $\mathbf{F}$, normal to the panel, is then given by

$\mathbf{F}=-\frac{1}{2} \rho_{\infty} C_{\mathrm{p}} S V_{\infty}^{2} \cdot \mathbf{n}$,

with $S$ being the surface area.

\section{Wing-body interaction problem}

Interrupting the wing at the fuselage intersection would result in a discontinuity in the vortex distribution which will not end at the symmetry plane. The discontinuity will lead to a strong vortex being generated at the root of the wing, that is not counteracted by its symmetric part thus influencing the neighboring body panels. This condition is also not realistic since a vortex at the midplane is not representative of the physical phenomena.

A possible solution is discussed by Rusak et al. [17] which entails extending the wing panels to the midplane. The extension of the panels will immediately solve the problem of the discontinuity at the midplane shedding no extra vortices within the body. The tangency condition is omitted from the wing panels within the body, and the collocation point is removed. This modification to describe the physics of the wing-body interaction is illustrated in Figs. 1, 2.

\section{Verification with literature}

The implementation has been validated against the work by Singh et al. [16], proving both analytical and experimental reference results on the model shown in Fig. 3a, with the wing located at $4.0 \mathrm{~m}$ along the fuselage symmetry line. The wing is rectangular with a $8.8-\mathrm{m}$ span and a $1.5-\mathrm{m}$ chord. An extensive description of the model is found in Singh et al. [16]. The results of the comparison are shown in Fig. 3, where the pressure coefficient is sampled at different radial positions $\theta$. The sign convention chosen for the radial position is shown in Fig. 3b.

The overall trends are in good agreement. There are two main differences between the model used in the present work and the one by Singh et al. [16] that ultimately cause the differences observed in the results. The differences are due to (1) the wing models used in the studies, and (2) the different models for the wing-body interaction. In particular,

- in the work by Singh et al. [16], the wing collocation points are defined on the wetted surface, with the panels modeling the 3D airfoil, whereas in the present work, the airfoil is reduced to its camber line thus not modeling the airfoil thickness. In the former case, the forces are thus perpendicular to the local airfoil wetted surface, as opposed to being perpendicular to the camber line (locally) as in the latter case. This explains the slight shift observed in the peak force;

- the wing-body interaction model used in the present work is not needed if the full 3D wing is modeled. In the numerical model in Singh et al., the wing panels will end at the junction meeting the fuselage panels. A discussion with regard to the differences with the experimental data presented in Singh et al. [16] has not been found.

\section{Full-aircraft aerodynamic model}

The aerodynamic models of wing, horizontal tail and fuselage are hereby summarized:

$$
\begin{cases}\mathbf{A}_{\mathrm{W}} \Gamma_{\mathrm{W}} & =\mathbf{B}_{\mathrm{W}} \\ \mathbf{A}_{\mathrm{HT}} \Gamma_{\mathrm{HT}} & =\mathbf{B}_{\mathbf{H}} T \\ \mathbf{A}_{\mathrm{F}} \sigma & =\mathbf{B}_{\mathbf{F}},\end{cases}
$$

Fig. 2 Fuselage model used for verfification

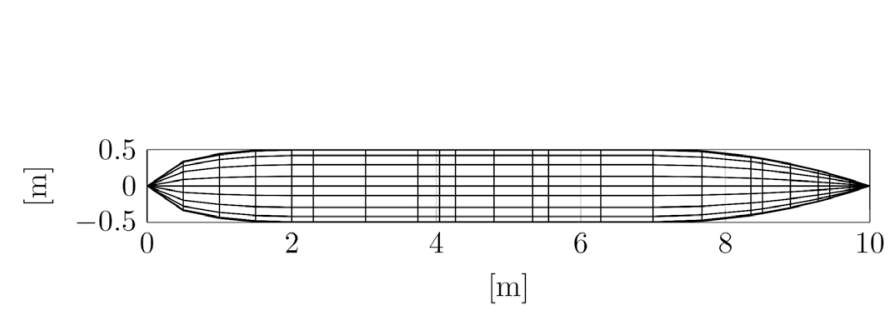

(a) Aerodynamic mesh

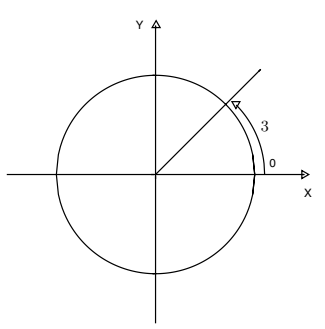

(b) Radial position $\theta$. 
Fig. 3 Fuselage $C_{p}$, in comparison with the work of Singh et al. [16]

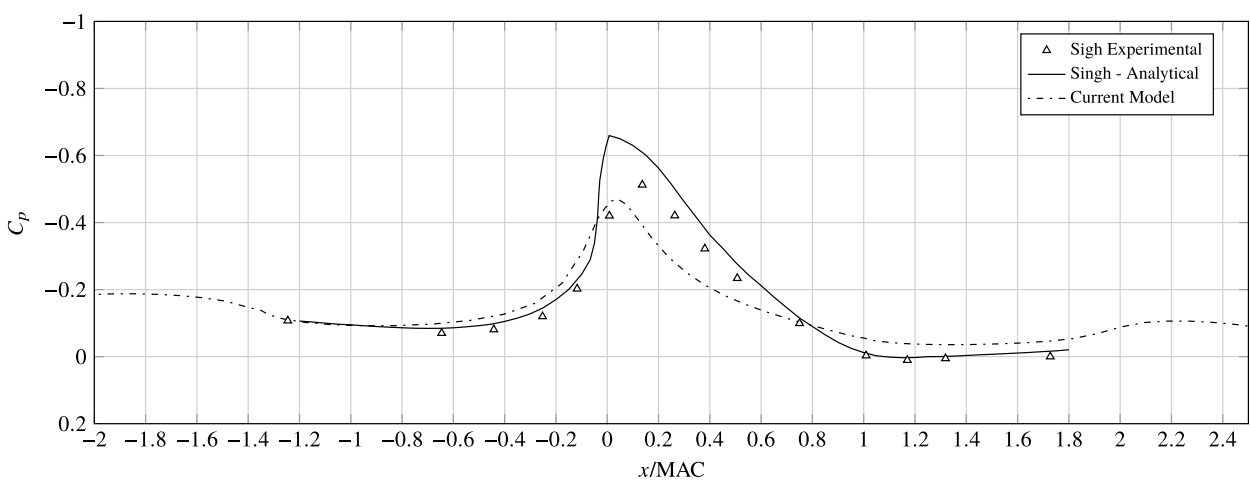

(a) Fuselage line at $\theta=+20 \mathrm{deg}$.

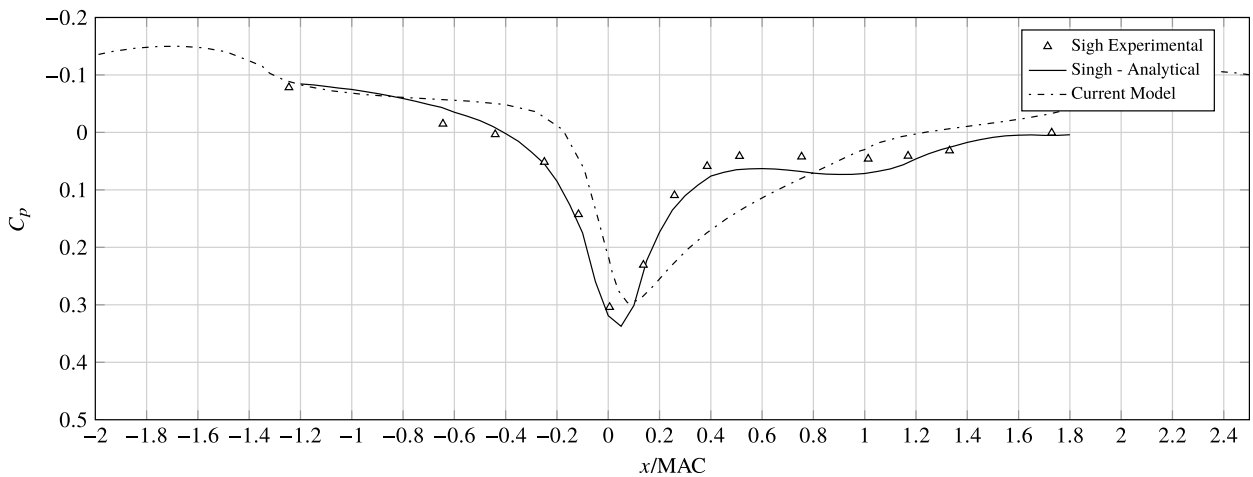

(b) Fuselage line at $\theta=-20 \mathrm{deg}$.

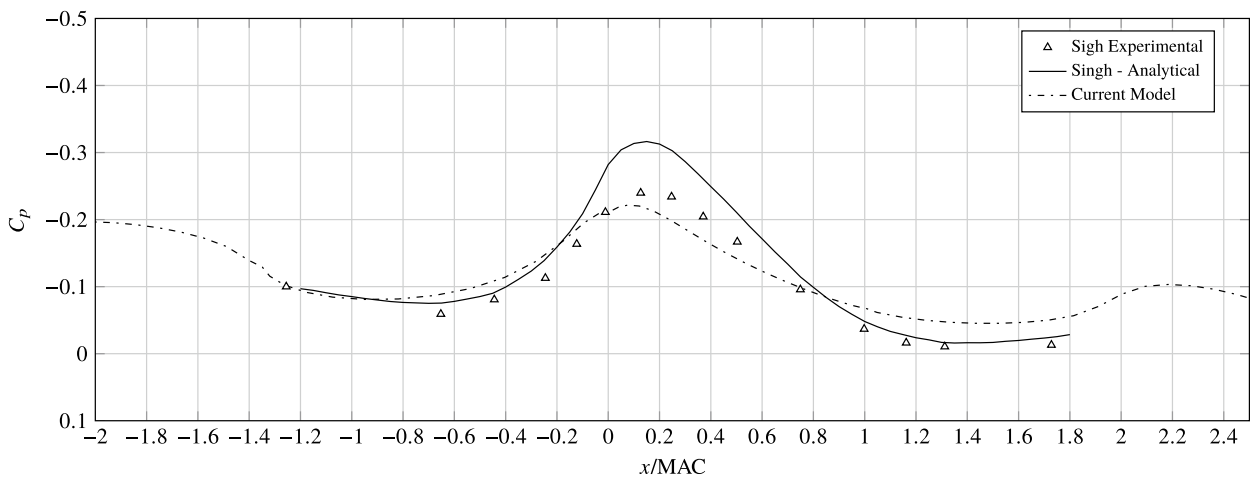

(c) Fuselage line at $\theta=+45 \mathrm{deg}$.

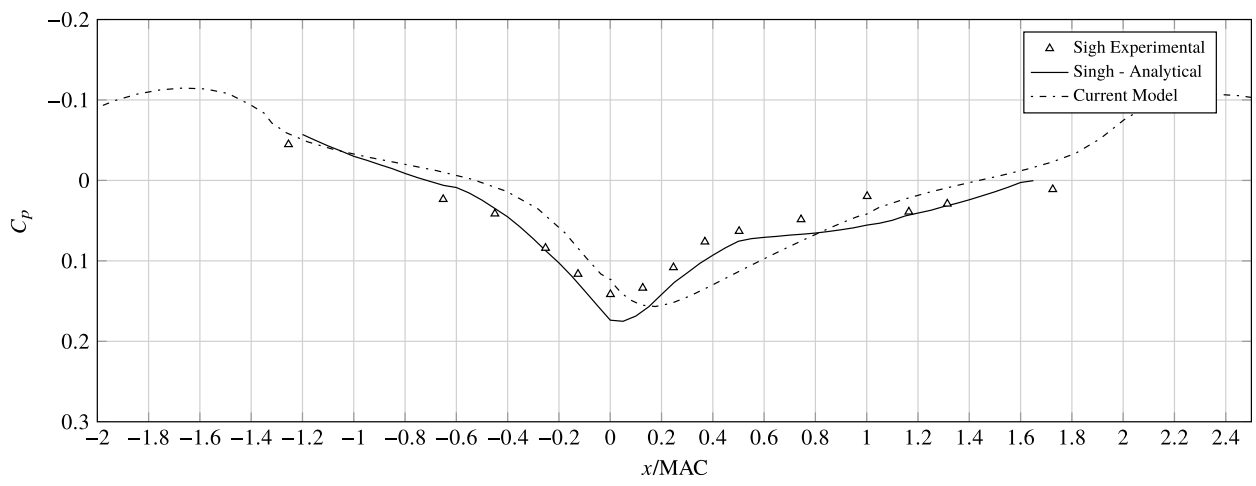

(d) Fuselage line at $\theta=-45 \mathrm{deg}$. 
with $\mathbf{A}$ being the aerodynamic influence coefficients of fuselage (F), wing (W) and horizontal tail (HT), $\sigma$ the fuselage source strength, and $\Gamma$ the vortex strength.

The derivation of the vortex-lattice models for wing and horizontal tail is extensively discussed in the work of Werter et al. [24, 25].

To couple the three equations and obtain the full-aircraft aerodynamic model, the following aerodynamic influence coefficients are needed, namely

- wing on fuselage, and horizontal tail $\left(\mathbf{A}_{\mathrm{W}, \mathrm{F}}, \mathbf{A}_{\mathrm{W}, \mathrm{HT}}\right)$,

- horizontal tail on fuselage, and wing $\left(\mathbf{A}_{\mathrm{HT}, \mathrm{F}}, \mathbf{A}_{\mathrm{HT}, \mathrm{W}}\right)$,

- fuselage on wing, and horizontal tail $\left(\mathbf{A}_{\mathrm{F}, \mathrm{W}}, \mathbf{A}_{\mathrm{F}, \mathrm{HT}}\right)$.

The system of equations can then be written as

$\left[\begin{array}{ccc}\mathbf{A}_{\mathrm{W}} & \mathbf{A}_{\mathrm{W}, \mathrm{HT}} & \mathbf{A}_{\mathrm{W}, \mathrm{F}} \\ \mathbf{A}_{\mathrm{HT}, \mathrm{W}} & \mathbf{A}_{\mathrm{HT}} & \mathbf{A}_{\mathrm{HT}, \mathrm{F}} \\ \mathbf{A}_{\mathrm{F}, \mathrm{W}} & \mathbf{A}_{\mathrm{F}, \mathrm{HT}} & \mathbf{A}_{\mathrm{F}}\end{array}\right]\left[\begin{array}{c}\Gamma_{\mathbf{W}} \\ \Gamma_{\mathbf{H}} T \\ \sigma\end{array}\right]=\left[\begin{array}{c}\mathbf{B}_{\mathrm{W}} \\ \mathbf{B}_{\mathbf{H}} T \\ \mathbf{B}_{\mathbf{F}}\end{array}\right]$.

Once the source and vortex strength distribution is known across all the panels, the forces are calculated. The force distribution along the fuselage is given by Eq. 13. For wing where $\Delta y_{i, j}$ is the width of the $i, j$ panel. The pressure difference is then given by

$\Delta p_{i, j}=\frac{L_{i, j}}{S_{i, j}}$,

with $S_{i, j}$ being the area of the panel.

\section{Static aeroelastic solution}

The fully non-linear aeroelastic solution is given by

$\left\{\begin{array}{l}\mathbf{F}_{s}=\mathbf{F}_{a}+\mathbf{F}_{\mathrm{ext}} \\ L=W \\ M_{\mathrm{CG}}=0\end{array}\right.$

where the subscripts $s, a$ and ext refer to the internal, aerodynamic and external forces, respectively.

The solution to the static problem is described by the displacement vector $\mathbf{p}$, the trim angle of attack $\alpha$ and the horizontal tail angle of attack $\delta$. Expanding the non-linear system with a first-order Taylor series with respect to $\mathbf{p}, \alpha$ and $\delta$, the system of equation can be written as

$$
\left\{\begin{array}{l}
\frac{\partial \mathbf{F}_{s}}{\partial \mathbf{p}} \Delta \mathbf{p}+\mathbf{F}_{s, 0}=\mathbf{F}_{a, 0}+\mathbf{F}_{\mathrm{ext}, 0}+\frac{\partial\left(\mathbf{F}_{a}+\mathbf{F}_{\mathrm{ext}}\right)}{\partial \mathbf{p}} \Delta \mathbf{p}+\frac{\partial\left(\mathbf{F}_{a}+\mathbf{F}_{\mathrm{ext}}\right)}{\partial \alpha} \Delta \alpha+\frac{\partial\left(\mathbf{F}_{a}+\mathbf{F}_{\mathrm{ext}}\right)}{\partial \delta} \Delta \delta \\
L_{0}+\frac{\partial L}{\partial \mathbf{p}} \Delta \mathbf{p}+\frac{\partial L}{\partial \alpha} \Delta \alpha+\frac{\partial L}{\partial \delta} \Delta \delta=\mathbf{W} \\
\mathbf{M}_{\mathrm{CG}, 0}+\frac{\partial \mathbf{M}_{\mathrm{CG}}}{\partial \mathbf{p}} \Delta \mathbf{p}+\frac{\partial \mathbf{M}_{\mathrm{CG}}}{\partial \alpha} \Delta \alpha+\frac{\partial \mathbf{M}_{\mathrm{CG}}}{\partial \delta} \Delta \delta=\mathbf{0},
\end{array}\right.
$$

and horizontal tail, the force is given by the Kutta-Jukowski theorem. With $i$ and $j$ being the spanwise and chordwise indexes, the lift expression for a single panel (identified with the indexes $i, j$ ) is given by

$L_{i, j}= \begin{cases}\rho V_{\infty} \Gamma_{i, j} \Delta y_{i, j}, & \text { if } j=1 \\ \rho V_{\infty}\left(\Gamma_{i, j}-\Gamma_{i, j-1}\right) \Delta y_{i, j}, & \text { if } j \neq 1,\end{cases}$ where the subscript 0 refers to the initial value of the forces.

It is important to notice that (1) the internal forces $\left(\mathbf{F}_{s}\right)$ do not depend on the angle of attack of the wing, nor the horizontal tail deflection, and (2) the weight is independent of all three state variables.

The linearized equations can be now written in matrix format. Remembering that the derivatives of the forces $\mathbf{F}_{s}$, $\mathbf{F}_{a}$ and $\mathbf{F}_{\text {ext }}$ with respect to $\mathbf{p}$ are nothing other than the definition of the stiffness matrices, the system can also be written as

$$
\left[\begin{array}{ccc}
\mathbf{K}_{s}-\left(\mathbf{K}_{a}+\mathbf{K}_{\mathrm{ext}}\right) & -\frac{\partial \mathbf{F}_{a}}{\partial \alpha}-\frac{\partial \mathbf{F}_{\mathrm{ext}}}{\partial \alpha} & -\frac{\partial \mathbf{F}_{a}}{\partial \delta}-\frac{\partial \mathbf{F}_{\mathrm{ext}}}{\partial \delta} \\
\frac{\partial \mathbf{L}}{\partial \mathbf{p}} & \frac{\partial \mathbf{L}}{\partial \alpha} & \frac{\partial \mathbf{L}}{\partial \delta} \\
\frac{\partial \mathbf{M}_{\mathrm{CG}}}{\partial \mathbf{p}} & \frac{\partial \mathbf{M}_{\mathrm{CG}}}{\partial \alpha} & \frac{\partial \mathbf{M}_{\mathrm{CG}}}{\partial \delta}
\end{array}\right]\left[\begin{array}{c}
\Delta \mathbf{p} \\
\Delta \alpha \\
\Delta \delta
\end{array}\right]=\left[\begin{array}{c}
\mathbf{F}_{a, 0}+\mathbf{F}_{\mathrm{ext}, 0}-\mathbf{F}_{s, 0} \\
\mathbf{W}-\mathbf{L}_{0} \\
-\mathbf{M}_{\mathrm{CG}, 0}
\end{array}\right]
$$

or in a more compact format

$$
\mathbf{J x}=\mathbf{R}_{0},
$$


Table 1 Details of constraint limits and margins of safety

\begin{tabular}{ll}
\hline Minimum thickness & 0.00183 \\
& {$[\mathrm{~m}](10$} \\
& plies $)$ \\
Maximum tensile strain & $6420[\mu \mathrm{s}]$ \\
Maximum compression strain & $4857[\mu \mathrm{s}]$ \\
Maximum shear strain & $5332[\mu \mathrm{s}]$ \\
MS for aeroelastic stability & 0.15 \\
Interval of local angle of attack & $\pm 15\left[^{\circ}\right]$ \\
MS of buckling factor & 0.4375 \\
Minimum aileron effectiveness & 0.10
\end{tabular}

where $\mathbf{x}^{T}=\{\mathbf{p}, \alpha, \delta\}, \mathbf{J}$ referred to as the Jacobian of the system, and $\mathbf{R}_{0}$ is the residual. The states of the trim condition can be thus calculated as

$\mathbf{x}=\mathbf{J}^{-1} \mathbf{R}_{0}$.

This approach is suitable for non-linear methods provided the stiffness matrices and force vectors are updated. For linear static aeroelasticity the system can be solved in a single iteration.

In case the configuration is not to be trimmed, the solution becomes trivial since $\Delta \alpha$ and $\Delta \delta$ are prescribed. The solution can be derived from the first line of Eq. 20 .

\section{Aeroelastic tailoring studies}

\subsection{Objective and constraints}

The objective of the optimizations is minimizing the structural mass of the composite wing by optimizing its thickness and stiffness distribution, described as a function of lamination parameters. As discussed in Sect. 1, the formulation in terms of lamination parameters is continuous and is thus suitable for gradient-based optimizers. The present work uses the globally convergent method of moving asymptotes (GCMMA) as presented by Svanberg [28].

The constraints adopted for the aeroelastic tailoring optimization and their relative margins of safety (MS) or limits are summarized in Table 1 . The margins of safety combine both certification aspects (according to CS25) and knockdown factors, with reference to the work of Kassapoglou [29].

\subsection{Optimization set-up}

The aircraft model used for the aeroelastic tailoring studies hereby discussed is based on the NSR ${ }^{1}$ benchmark aircraft. The geometric description of the wing and the non-structural mass, taken into account for the purposes of these studies,

\footnotetext{
${ }^{1}$ NSR new short range.
}

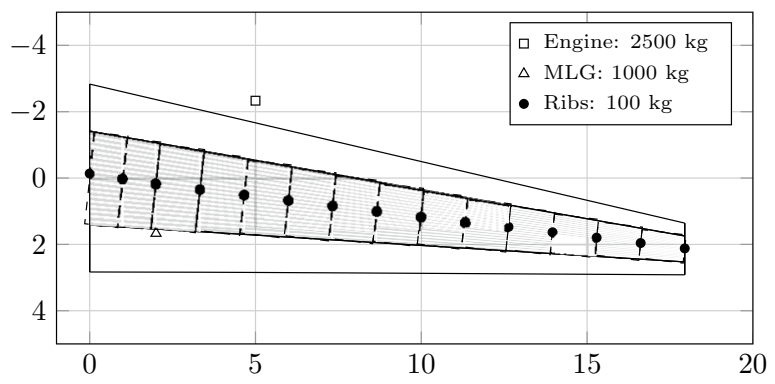

Fig. 4 Details of the NSR wing model. $M L G$ main landing gear

Table 2 Overview of static load cases

\begin{tabular}{llllll}
\hline LC & Mach $[-]$ & EAS $[\mathrm{m} / \mathrm{s}]$ & $\mathrm{H}[\mathrm{m}]$ & $\mathrm{n}_{z}$ & Description \\
\hline 1 & 0.75 & 252.50 & 6700 & 1 & Dive speed \\
2 & 0.70 & 192.47 & 6700 & 2.5 & Pull-up maneuver \\
3 & 0.70 & 192.47 & 6700 & -1 & Push-down maneuver \\
\hline
\end{tabular}

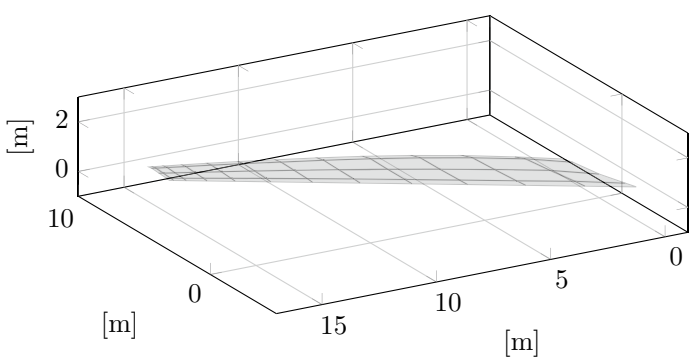

Fig. 5 Wing aerodynamic model (ID $=0)$

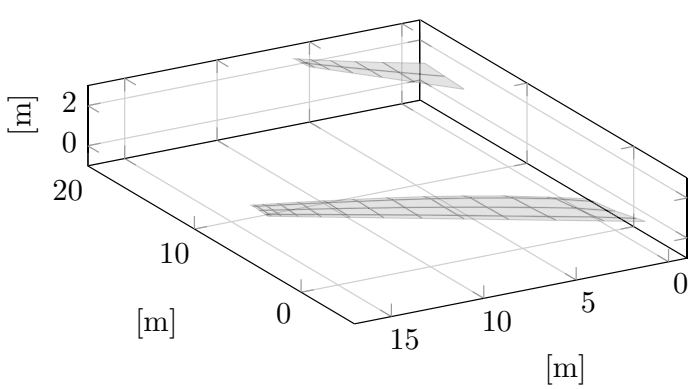

Fig. 6 Wing-tail aerodynamic model (ID = 1)

are shown in Fig. 4. The loadcases adopted for the study can be found in Table 2.

The NSR aircraft has been modeled with a flexible composite wing, a rigid horizontal tail and rigid fuselage. At the start of the optimization, the wing has a zero-dominated layup (60\% in $0,10 \%$ in 90 and $30 \%$ in \pm 45 ) for top and bottom skin and quasi-isotropic (QI) spars. Such stiffness 


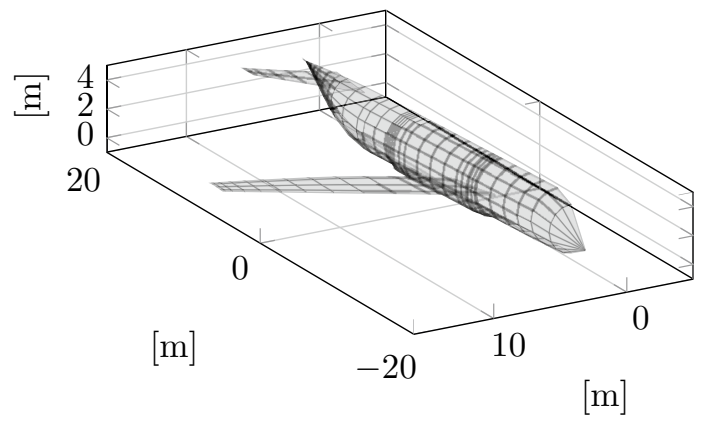

Fig. 7 Aircraft aerodynamic mode $(\mathrm{ID}=2)$

distribution is widely used as a reference in aeroelastic tailoring, as discussed in Dillinger et al. [23], for more realistic estimation of the weight-saving potential of aeroelastic tailoring as opposed to a QI initial design which is highly over-designed.

Three aeroelastic tailoring optimizations have been run on the NSR-wing model: (1) using a wing aerodynamic model as shown in Fig. 5, (2) a wing-tail aerodynamic model, Fig. 6 and (3) a full-aircraft aerodynamic description as in Fig. 7. The design variables for each of the three optimizations are the lamination parameters and the thickness of the composite wing. The goal of the study is to observe the difference in optimum design due to a change in loads because of (1) the moment equilibrium due to the horizontal tail, and (2) the extra lift generated by the fuselage.

\subsection{Optimization results}

The wing-only optimization (ID $=0$ ) converged to a final structural mass of $1700 \mathrm{~kg}$, which will be used as a reference for this discussion. As shown in Table 3, the wing-tail (ID $=1)$ is $2.5 \%$ lighter, whereas the aircraft $(\mathrm{ID}=2)$ shows an increase in structural mass of approximately $11 \%$. The convergence history of the objective function is shown in Fig. 8. These results can be explained by looking at the physics behind aeroelastic tailoring, and some of the results shown in Appendices A through C.

Fuselage influence The first effect of the fuselage aerodynamic model is a drop of lift at the root of the wing, namely the area at the midline that lies within the fuselage. Since the aircraft is to remain trimmed at a given flight point, the wing has to generate more lift, hence its resultant will shift outboard. For a swept back wing, an outboard shift of the lift resultant also implies that its application point moves towards the rear of the aircraft. The shift of the aerodynamic center alters its relative position with respect to the center of gravity, thus changing the dynamics of the whole aircraft.

The fuselage is also responsible for inducing a relatively large nose-up moment (assumed positive in this paper). The
Table 3 Converged objective in comparison

\begin{tabular}{llll}
\hline ID & Model & Mass $[\mathrm{kg}]$ & $\Delta[\%]$ \\
\hline 0 & Wing-only aerodynamics & 1700 & N/A \\
1 & Wing-tail aerodynamics & 1602 & -2.5 \\
2 & Aircraft aerodynamics & 1828 & +11 \\
\hline
\end{tabular}

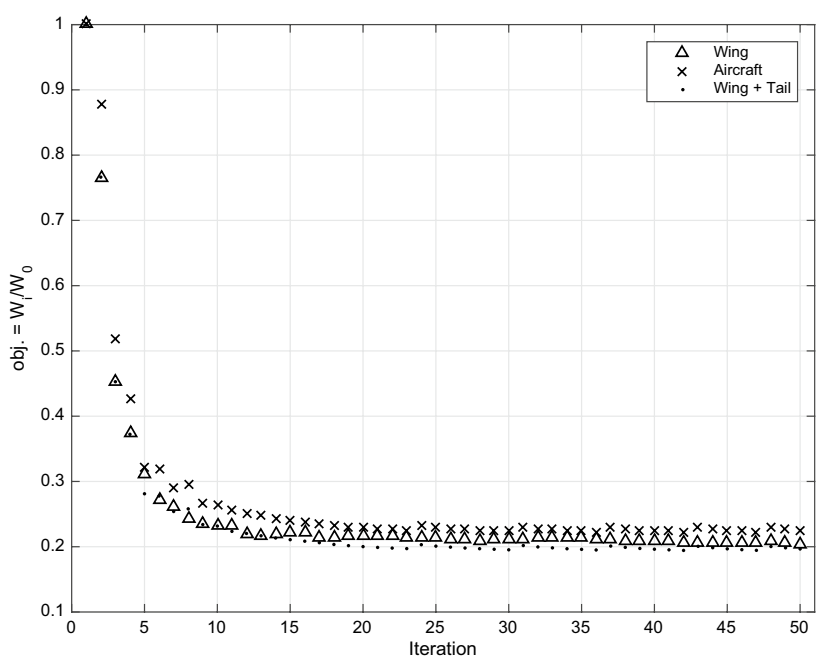

Fig. 8 Convergence of the objective functions

reason behind the introduction of this moment has to do with the shape of the fuselage and its rotational asymmetry. This nose-up moment is important for two reasons:(1) it affects the lift distribution significantly since it plays an important role in the moment equation, (2) the pitching moment is unstable as discussed in the work by Singh et al. [16].

Horizontal tail influence The influence of the tail is particularly on the moment equilibrium and it depends on the relative position between the wing aerodynamic center (AC) and the aircraft center of gravity (CG).

In case the $\mathrm{CG}$ is in front of the $\mathrm{AC}$, the horizontal tail generates a negative lift to balance the moment. As a result, the lift the wing has to generate is equal to the weight plus the lift generated by the tail:

$L_{\text {wing }}=W+L_{\text {tail }}$;

hence,

$L_{\text {wing }}>W$.

The opposite scenario, where the $\mathrm{AC}$ is in front of the CG, the horizontal tail generates positive lift; thus,

$L_{\text {wing }}<W$.

Combined effects The effects caused by the fuselage and tail individually are to be weighted against the effect the practices of aeroelastic tailoring have on the aircraft. The 
objective of the optimization via aeroelastic tailoring is weight minimization that is usually achieved by shifting the lift inboard and reducing the root bending moment at highload factors. For a swept back wing, an inboard shift of the lift resultant also implies a forward shift of the aerodynamic center. At the same time, since the structural mass of the wing is descreasing, the CG is moving aft, towards the rear of the aircraft.

In optimization 1 (wing-tail aerodynamics), the configuration where the $\mathrm{AC}$ is in front of the $\mathrm{CG}$ is reached as a consequence of weight minimization and load alleviation due to aeroelastic tailoring. This explains the lower structural mass (approximately $2 \%$ compared to the wing reference) since at a given flight points, the loads on the wing are reduced.

The physics is different for optimization 2 (full-aircraft aerodynamics). Due to the high nose-up moment induced by the fuselage, and the drop of lift at the midline, the AC remains aft of the center of gravity thus explaining the increase in lift generated by the wing. The overall increase in the stress state in the wing explains the increase of the optimum structural mass up to app. $11 \%$.

Tailoring The results on stiffness and thickness tailoring are summarized in Appendix A. In all three optimization cases, we can see the following trends:

- a beneficial wash-out effect is induced by controlling the stiffness and thickness distribution. An example of wash-out control through thickness design is observed in the spar thickness distribution in Fig. 11 in Appendix A where the front spar is thicker than the rear spar. This allows the rear spar to deform more, compared to the front spar, and thus reduce the local angle of attack. The stiffness tailoring, on the other hand, controls the local twist distribution by rotating the principal direction of the design patches. This phenomenon can be observed in the optimized stiffness distribution of both top and bottom skin in Figs. 9 and 10 in Appendix A.

- the optimized stiffness distribution is a result of the particular active constraint associated with every patch. For example, the patches where the buckling failure index is closer to the critical value of 1.0, the optimized stiffness approaches almost a cross-ply stiffness distribution that is most suitable for a buckling driven design. On the other hand, a very defined principal direction is typical of patches where the strain failure index is critical.

Margins This paragraph discusses the results in terms of margins calculated during the optimization via aeroelastic tailoring. An overview of the strain and buckling failure indexes of the design configurations used for this study is shown in Appendices B and C. Both measures are conservative to account for material imperfections, damage and scatter.
Table 4 Overview of aileron effectiveness

\begin{tabular}{llll}
\hline LC & Wing-only & Wing-tail & Full-aircraft \\
\hline 1 & $0.150^{\mathrm{a}}$ & $0.150^{\mathrm{a}}$ & $0.150^{\mathrm{a}}$ \\
2 & 0.183 & 0.185 & 0.164 \\
3 & 0.190 & 0.187 & 0.169 \\
\hline
\end{tabular}

${ }^{\mathrm{a}}$ Constraint is active. The lower bound of aileron is set at 0.150

The outcome of the optimization shows two main trends regarding the sizing constraints for the final design, shown in Fig. 12 on Appendix B, namely:

- aileron effectiveness, found in Table 4, is active in the load case at dive speed (LC1),

- buckling and/or strains are active in the maneuver loadcases (LC2, LC3).

The aileron effectiveness constraint is active under highspeed loading conditions at $1 \mathrm{~g}$. The lower bound set to the constraint, namely 0.15 , is a very conservative measure and can vary depending on the type of aircraft and mission. The interesting trade-off is to compare the wing designed for a minimum level of aileron effectiveness within the flight envelope, to a wing where the aileron is actively controlled also beyond reversal.

The strain and buckling failure indexes are active in maneuver loadcases. The failure indexes are influenced by the internal load distribution, and consequently both the material and stiffness distribution of the optimized design will determine whether strain or buckling failure occurs first. In particular:

- in optimization 1, we see that the wing has more severe strain buckling failure indices compared to optimization 0 . This means that the in-plane stiffness distribution induces more wash-out to control the local twist distribution as a result of the higher deformations, whereas more patches have the out-of-plane stiffness closer to a cross-ply distribution to avoid buckling.

- in optimization 2, the wing is less critical in buckling and mainly sized by strain failure. This happens because the drop of lift at the root caused by the presence of the fuselage induces higher strains on the wing. As a result, the principal direction is aligned with the wing trailing edge for enhanced stiffness while the cross-ply distributions are less pronounced. 


\section{Conclusions}

The recent progress in aeroelasticity, in terms of both fundamental research and numerical solutions, has allowed the development of novel integrated frameworks to describe the tailoring optimization problem at aircraft level for more complete analyses compared to the classic aeroelastic tailoring solutions developed for a clamped wing. The assumptions and methodologies behind the classic aeroelastic tailoring practices are valid within certain levels of flexibility such that the interactions between the different parts of the aircraft are negligible.

The present work builds upon the aeroelastic tailoring framework developed by De Breuker et al. [12] to study the effects of a full-aircraft aerodynamic model on a tailored composite wing. This paper has shown the importance of a representative load description on the aeroelastic tailored design. The changes in lift distribution, center of gravity and aerodynamic center are driving the optimizer in different design regions with changes up to $11 \%$ in the mass of the final design.

It is important to realize that the changes in lift distribution are caused by the process of aeroelastic tailoring itself. By operating on the mass and stiffness properties of the wing, the load distribution changes in each iteration of the optimization loop. This also implies that both the center of gravity and the aerodynamic center are moving during the optimization, a phenomenon that has implication of the overall aircraft stability.

These observations will surely be of relevance in a future integrated approach to aeroelastic tailoring for composite aircraft design since they will affect other disciplines, for example, the flight dynamic performance, maneuverability and controllability of the aircraft.

The integrated approach for aircraft aeroelasticity and aeroelastic tailoring serves as a tool for design selection in the preliminary design phase. This concept is found already in the early work by Weisshaar et al. [2] where aeroelastic tailoring is recognized as an effective tool to scan the design space and select the best candidates for the subsequent phases. More applications of aeroelastic tailoring practices will be made feasible by development of both more efficient high-fidelity solutions, especially from the computational standpoint, and modular multidisciplinary frameworks for design purposes.

Acknowledgements The authors would like to thank the Clean Sky Joint Undertaking and the European Union for the support of SFWA which has been funded within the scope of the Seventh Framework Programme for Research and Technology by the European Commission through the Grant Agreement CSJU-GAM-SFWA-2008-001.

Open Access This article is distributed under the terms of the Creative Commons Attribution 4.0 International License (http://creativeco mmons.org/licenses/by/4.0/), which permits unrestricted use, distribution, and reproduction in any medium, provided you give appropriate credit to the original author(s) and the source, provide a link to the Creative Commons license, and indicate if changes were made.

\section{Appendix A: Optimized design variables}

See Figs. 9, 10, 11 here. 
Fig. 9 Optimized stiffness and thickness distribution of the top skin. A, in-plane stiffness; $\mathrm{D}$, out-of-plane stiffness; $t$, thickness

\section{Top Skin}
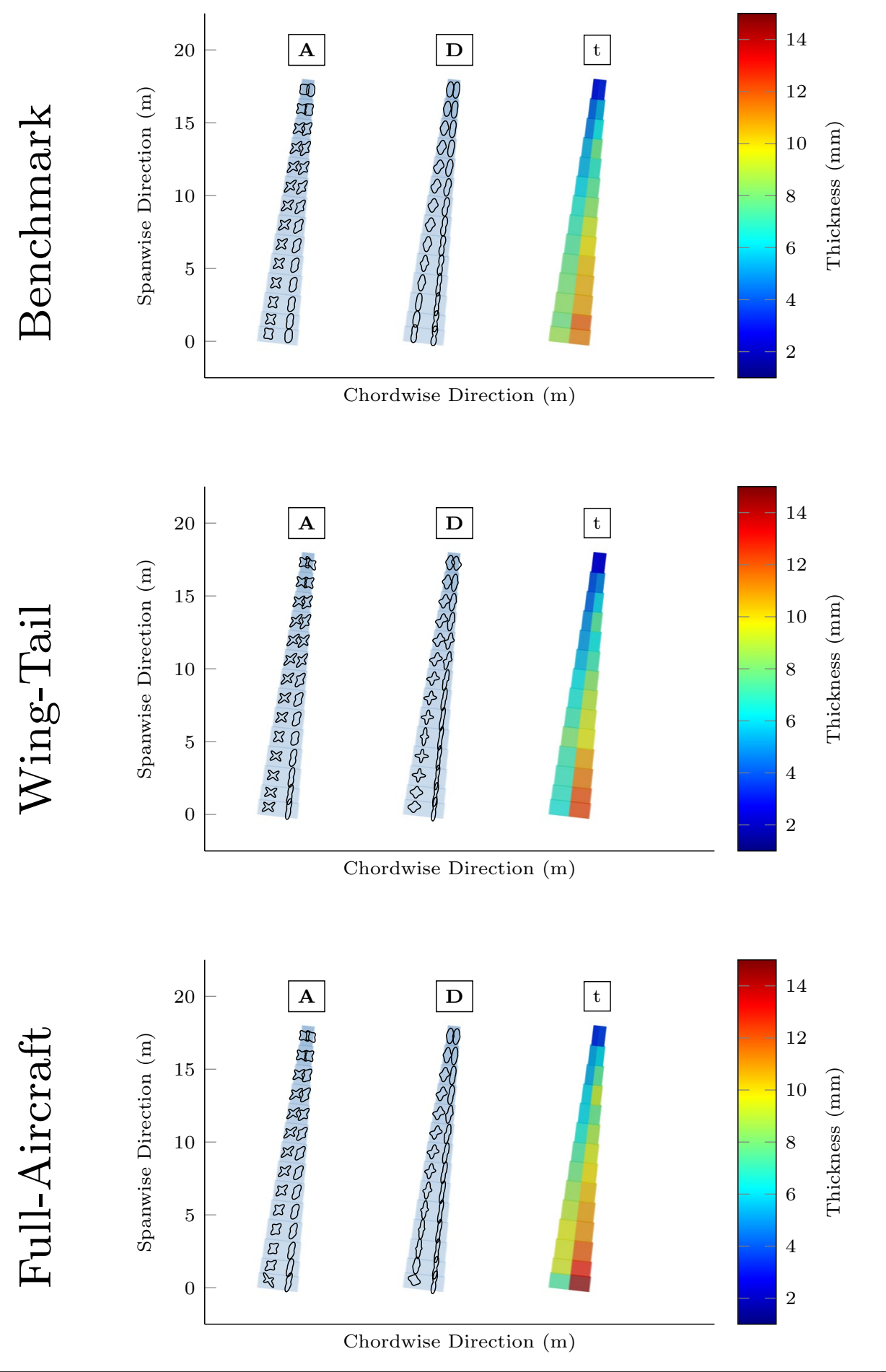
Fig. 10 Optimized stiffness and thickness distribution of the top skin. A, in-plane stiffness; $\mathrm{D}$, out-of-plane stiffness; $t$, thickness

\section{Bottom Skin}
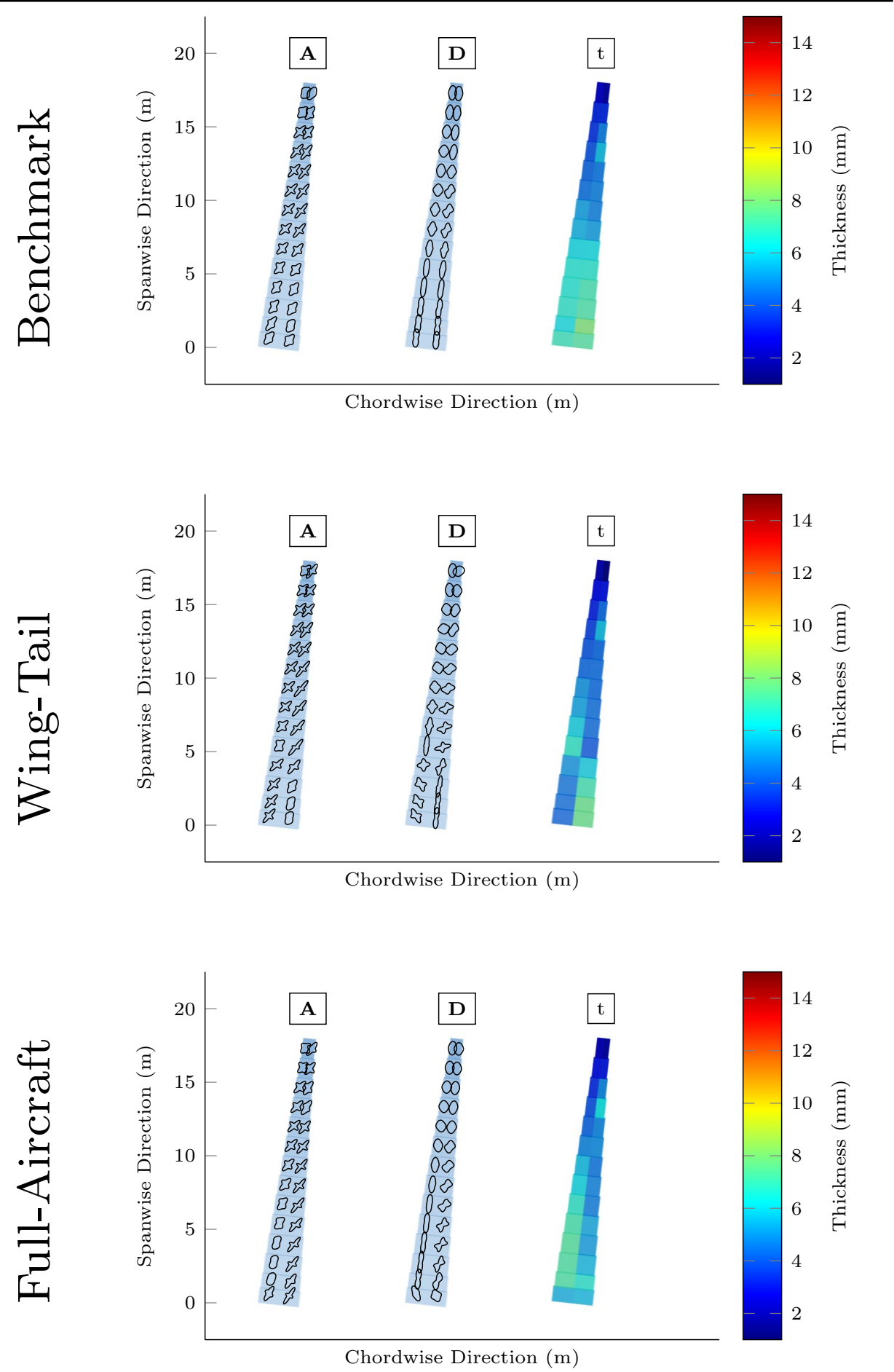
Fig. 11 Optimized stiffness and thickness distribution of the top skin. A, in-plane stiffness; $\mathrm{D}$, out-of-plane stiffness; $t$, thickness

\section{Spars}

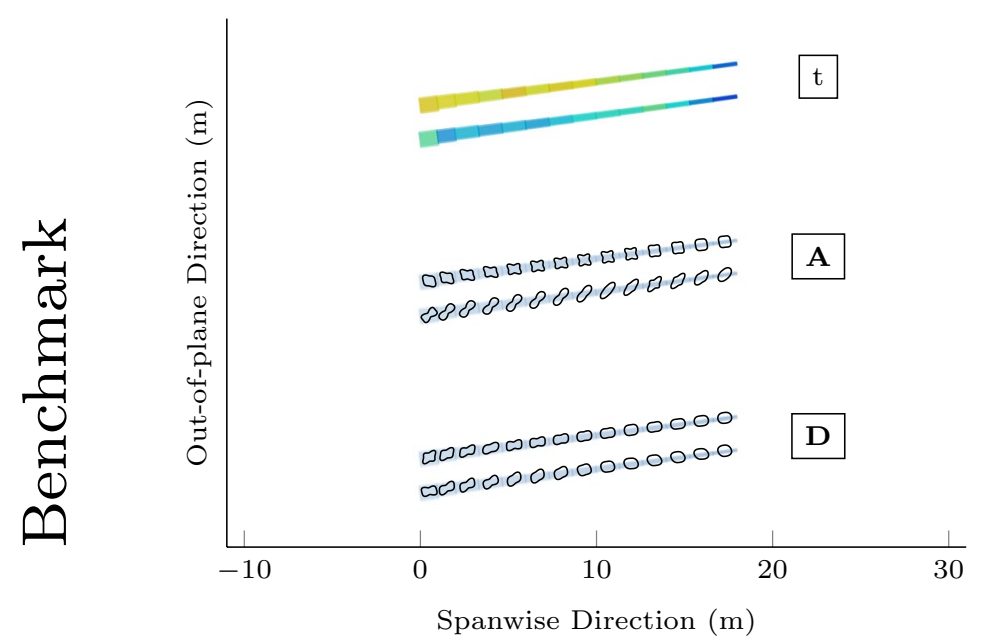

14

12

10

छ્छ

8

离

6

E

4

2
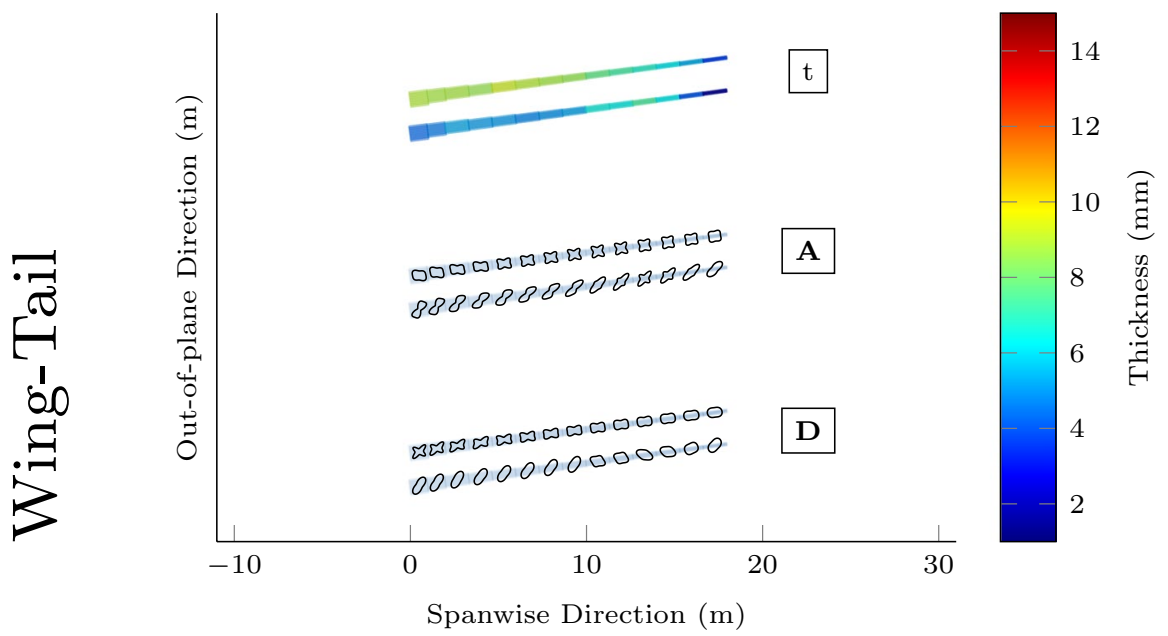

Spanwise Direction (m)

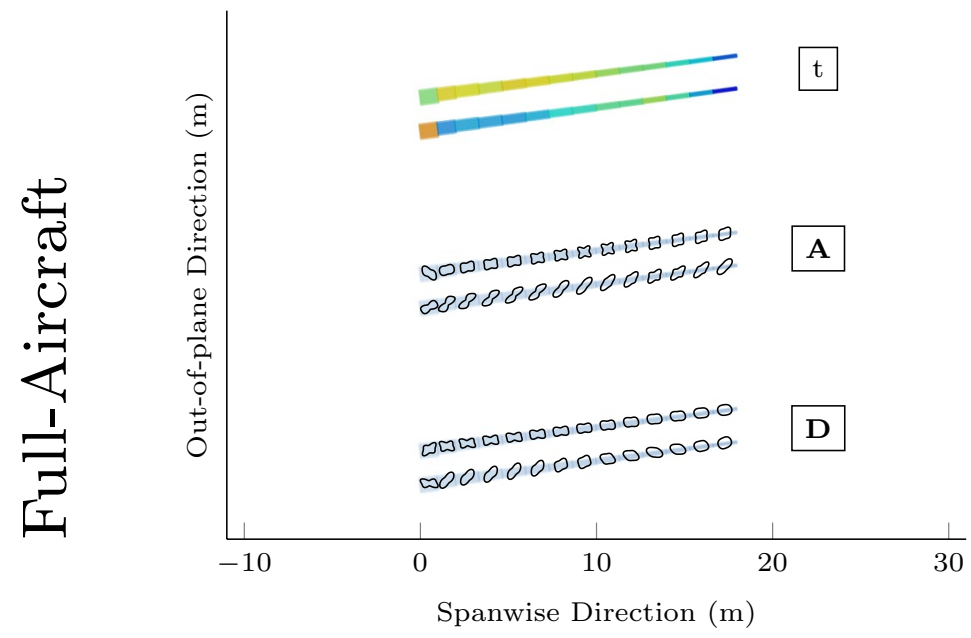




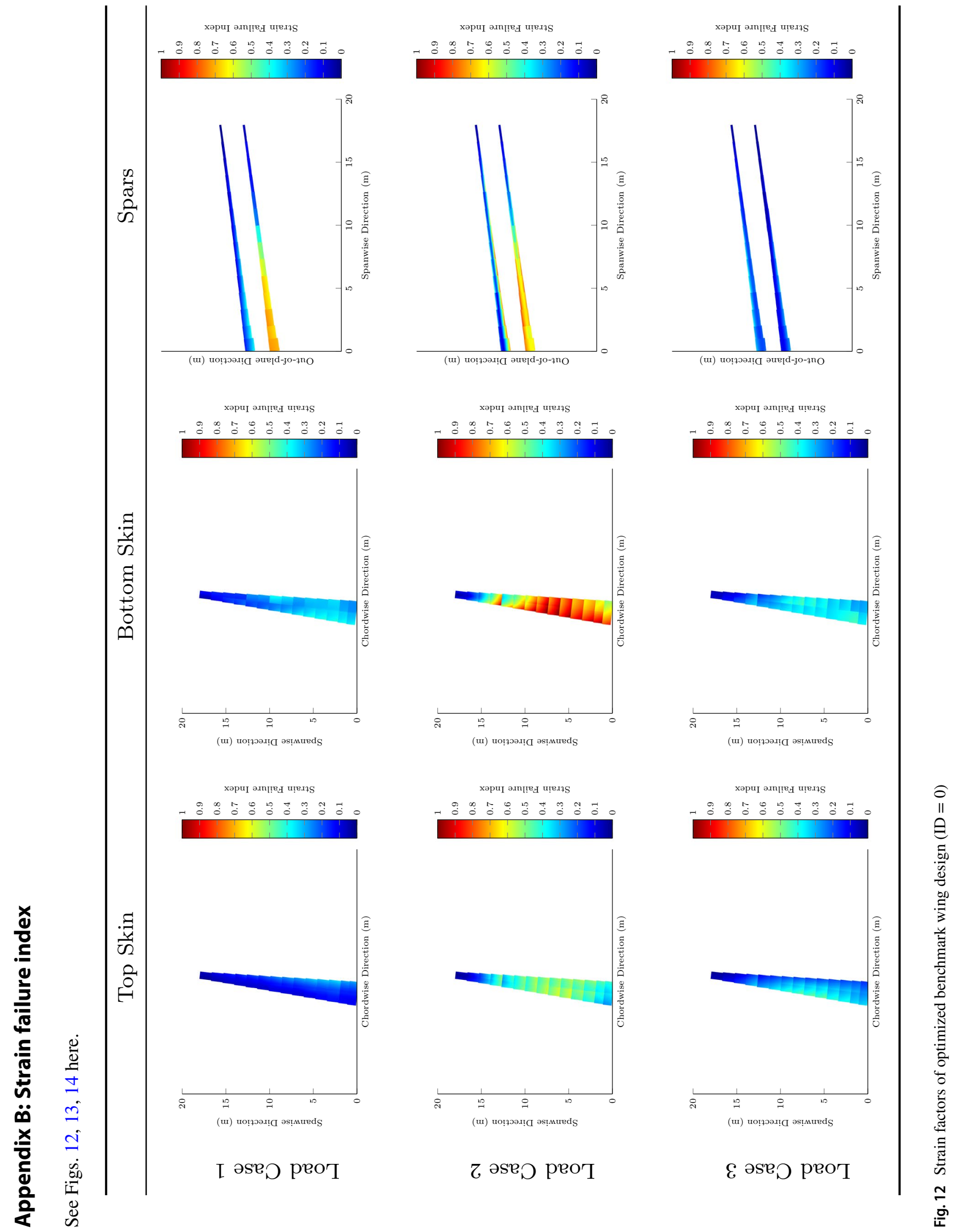




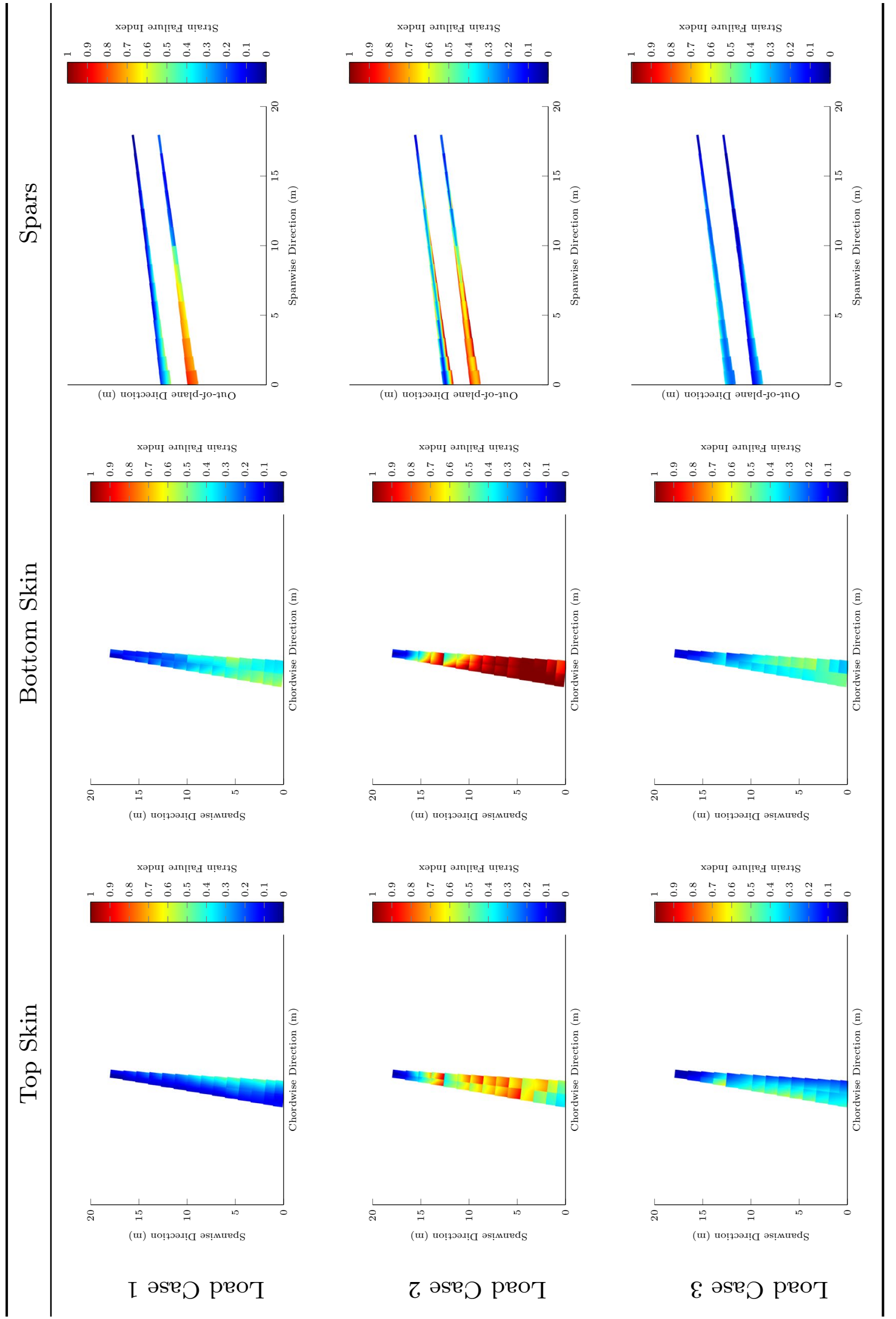

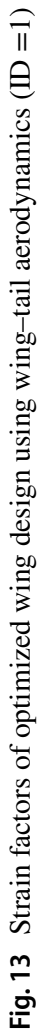




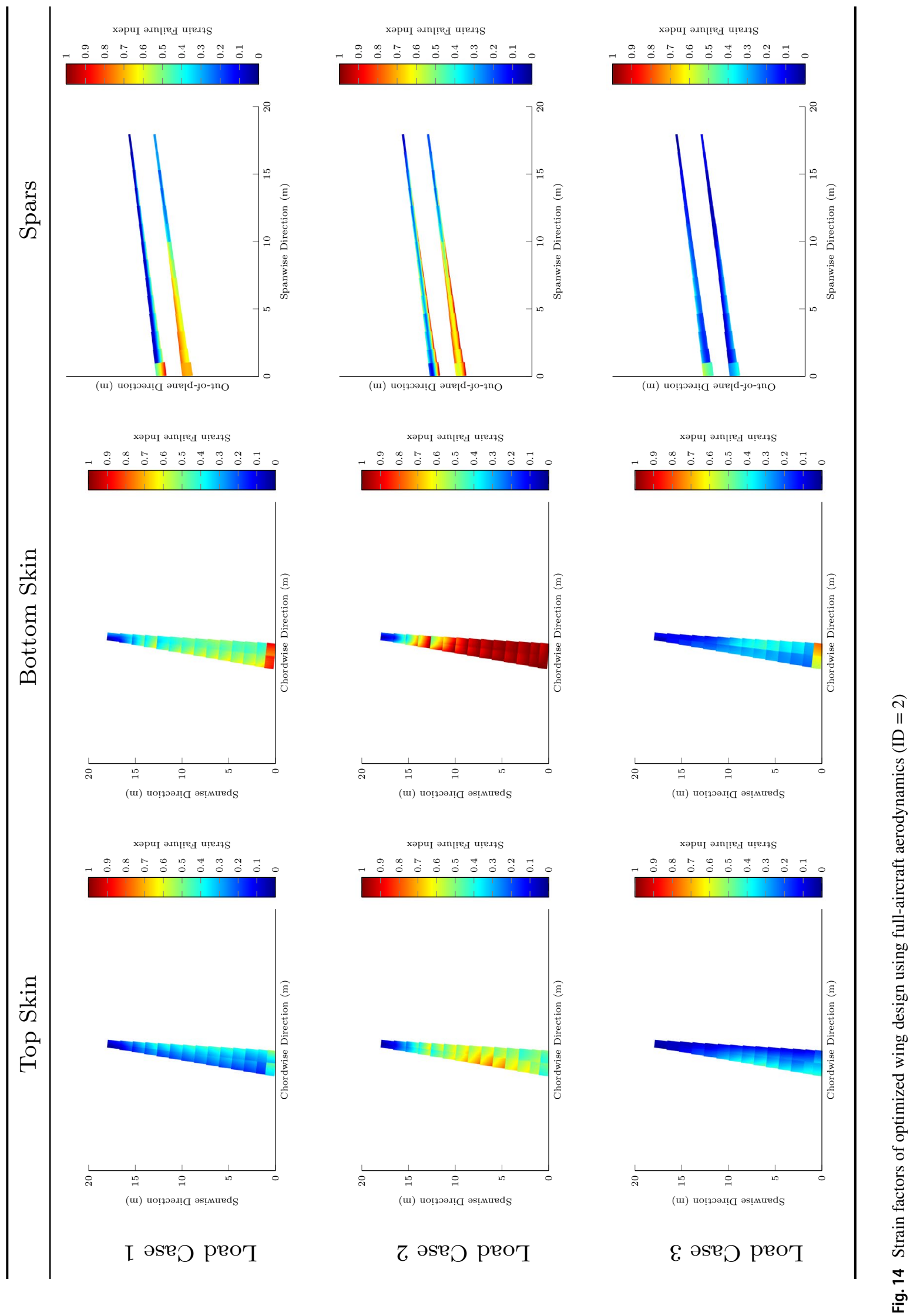




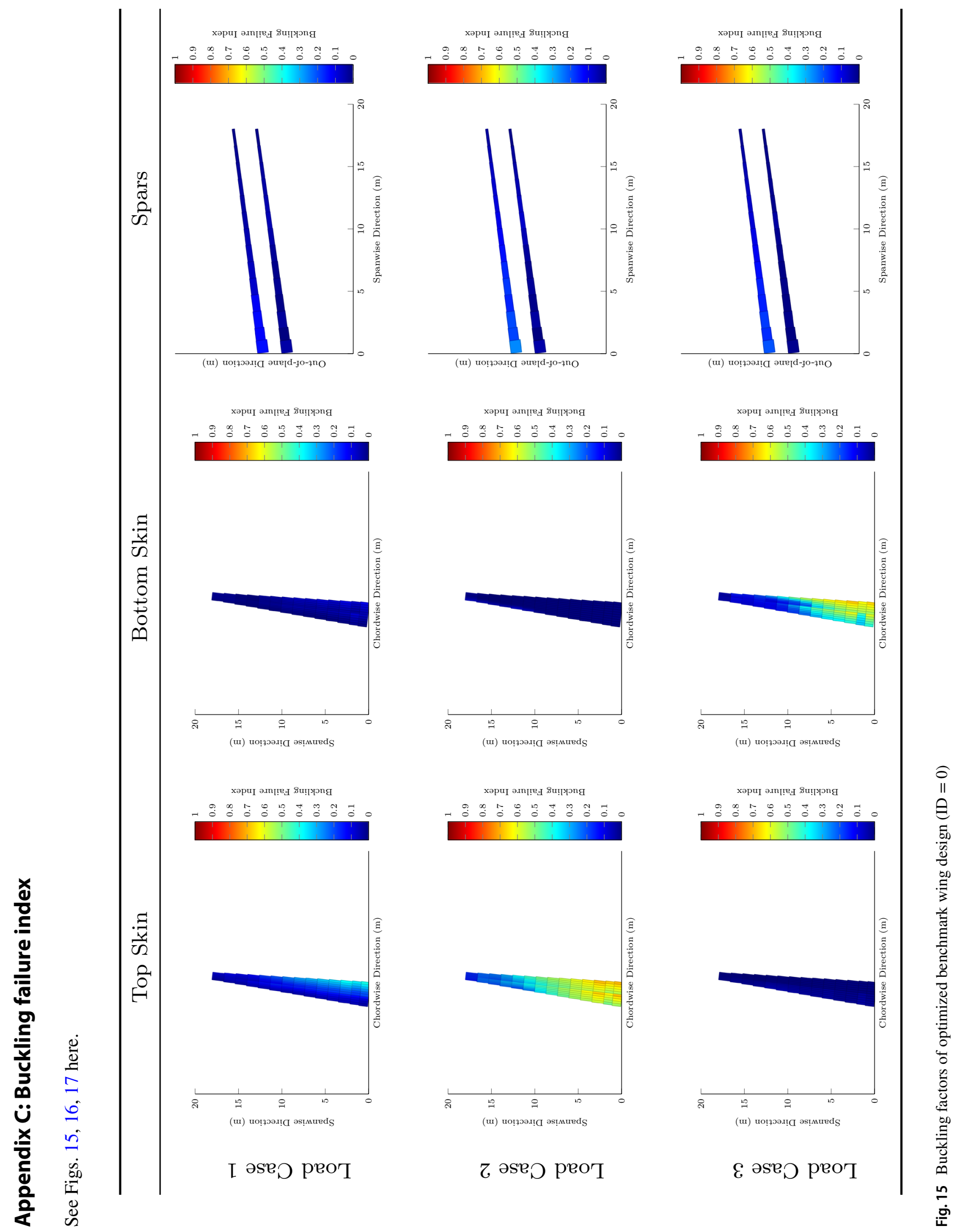




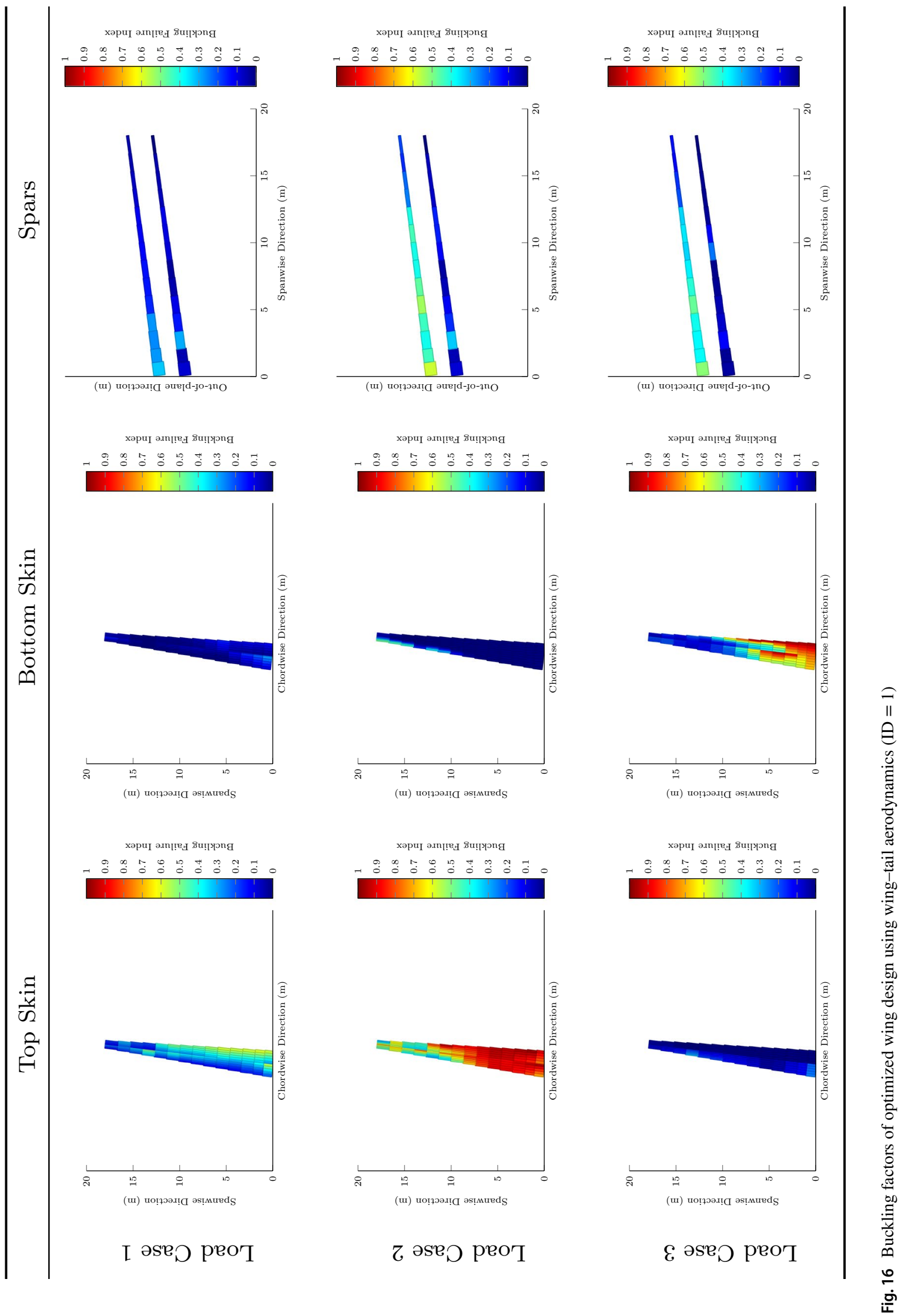




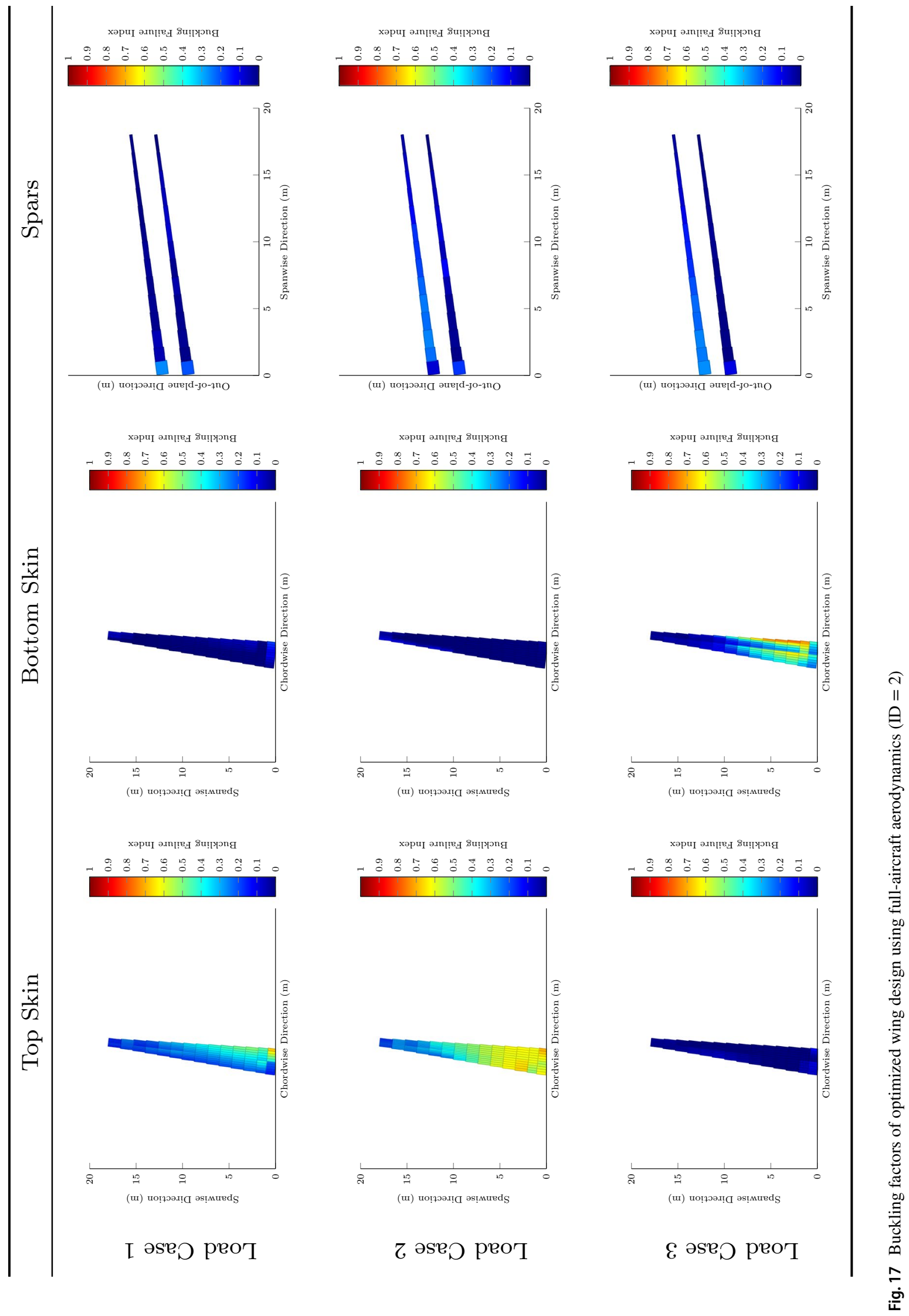




\section{References}

1. Shirk, M.H., Hertz, T.J., Weisshaar, T.A.: Aeroelastic tailoringtheory, practice and promise. J. Aircr. 23(1), 6-18 (1987)

2. Weisshaar, T.A.: Aeroelastic tailoring - creative use of unusual materials, AIAA 87-0976-CP, AIAA/ASME/ASCE/AHS 28th structures, structural dynamics and material conference, Monterey (1987)

3. Stanford, B.K., Wiesman, C.D., Jutte, C.V.: Aeroelastic tailoring of transport wings including transonic flutter constraints, 56th AIAA/ASCE/AHS/ASC structures, structural dynamics, and materials conference, AIAA SciTech Forum. https://doi. org/10.2514/6.2015-1127 (2015)

4. Stodieck, O., Cooper, J., Weaver, P., Kealy, P.: Aeroelastic tailoring of a representative wing-box using tow-steered composites. AIAA J. 2016, 1425-1439 (2016). https://doi.org/10.2514/1.J0553 64

5. Georgiou, G., Vio, G.A., Cooper, J.E.: Aeroelastic tailoring and scaling using bacterial foraging optimisation. Struct. Multidiscip. Optim. 50(1), 8199 (2014). https://doi.org/10.1007/s0015 8-013-1033-3

6. Manan, A., Vio, G.A., Harmin, M.Y., Cooper, J.E.: Optimization of aeroelastic composite structures using evolutionary algorithms. Eng. Optim. 42(2), 171184 (2010). https://doi.org/10.1080/03052 150903104358

7. Guo, S.J., Bannerjee, J.R., Cheung, C.W.: The effect of laminate lay-up on the flutter speed of composite wings. Proc. Inst. Mech. Eng. Part G J. Aerosp. Eng. 217(3), 115122 (2003). https://doi. org/10.1243/095441003322297225

8. Haddadpour, H., Zamani, Z.: Curvilinear fiber optimization tools for aeroelastic design of composite wings. J. Fluids Struct. 33, 180190 (2012). https://doi.org/10.1016/j.jfluidstructs.2012.05.008

9. Eastep, F.E., Tischler, V.A., Venkayya, V.B., Khot, N.S.: Aeroelastic tailoring of composite structures. J. Aircr. 36(6), 1041-1047 (1999)

10. Kim, T.U., Hwang, I.H.: Optimal design of composite wing subjected to gust loads. Comput. Struct. 83(19-20), 1546-1554 (2005)

11. Tian, W., Yang, Z., Gu, Y., Ouyang, Y.: Aeroelastic tailoring of a composite forward-swept wing using a novel hybrid pattern search method. J. Aerosp. Eng. 29(6), 04016056 (2016)

12. De Breuker, R., Abdalla, M.M., Gürdal, Z.: A generic morphing wing analysis and design framework. J. Intell. Mater. Syst. Struct. 22(10), 1025-1039 (2011)

13. Kameyama, M., Fukunaga, H.: Optimum design of composite plate wings for aeroelastic characteristics using lamination parameters. Comput. Struct. 85(3-4), 213224 (2007). https://doi. org/10.1016/J.Compstruc.2006.08.051

14. Jin, P., Song, B., Zhong, X., Yu, T., Xu, F.: Aeroelastic tailoring of composite sandwich panel with lamination parameters. Proc. Inst. Mech. Eng. Part G J. Aerosp. Eng. 230(1), 105-117 (2016). https://doi.org/10.1177/0954410015587724
15. Dillinger, J.K.S., Klimmek, T., Abdalla, M.M., Gurdal, Z.: Stiffness optimization of composite wings with aeroelastic constraints. J. Aircr. 50(4), 1159-1168 (2013). https://doi.org/10.2514/1. C032084

16. Singh, N., Aikat, S., Basu, B.C.: Incompressible potential flow about complete aircraft configurations. Aeronaut. J. 93(929), 335343 (1989)

17. Rusak, Z., Wasserstrom, E., Seginer, A.: Numerical calculation of nonlinear aerodynamics of wing-body configurations. AIAA J. 21(7), 929936 (1983)

18. Werter, N., De Breuker, R., Abdalla, M.: Continuous-time statespace unsteady aerodynamic modeling for efficient loads analysis. AIAA J. https://doi.org/10.2514/1.J056068 (2017)

19. Willaert, M., Abdalla, M., Gürdal, Z.: A simple finite element cross-sectional modeling of thin-walled beams. In: Proceedings of the 2nd aicraft structural design conference (2010)

20. Ferede, E., Abdalla, M.: Cross-sectional modelling of thin-walled composite beams. In: 55th AIAA/ASME/ASCE/AHS/ASC structures, structural dynamics, and materials conference, 10.2514 (2014)

21. Ijsselmuiden, S.T.: Optimal design of variable stiffness composite structures using lamination parameters. Ph.D. thesis, Delft University of Technology, Delft, The Netherlands (2011)

22. Khani, A., Ijsselmuiden, S.T., Abdalla, M.M., Gurdal, Z.: Design of variable stiffness panels for maximum strength using lamination parameters. Compos. Part B Eng. 42(3), 546552 (2011). https ://doi.org/10.1016/j.compositesb.2010.11.005

23. Dillinger, J.K.S.: Static aeroelastic optimization of composite wings with variable stiffness laminates. PhD Dissertation, Delft University of Technology (2014)

24. Werter, N., De Breuker, R.: A novel dynamic aeroelastic framework for aeroelastic tailoring and structural optimisation. Compos. Struct. 158, 369-386 (2016)

25. Werter, N., De Breuker, R., Abdalla, M.: Continuous-time statspace unsteady aerodynamic modelling for efficient aeroelastic load analysis. In: International Forum on Aeroelasticity and Structural Dynamics, June 28-July 2, 2015. Saint Petersburg, Russia (2015)

26. Hess, J.L., Smith, A.M.O.: Calculation of potential flow about arbitrary bodies. Progr. Aeronaut. Sci. 8, 1138 (1967)

27. Katz, J., Plotkin, A.: Low-Speed Aerodynamics. Mac Graw-Hill, New York (1991)

28. Svanberg, K.: The method of moving asymptotes a new method for structural optimization. Int. J. Numer. Methods Eng. 24, 359373 (1987)

29. Kassapoglou, C.: Design and Analysis of Composite Structures with Applications to Aerospace Structures. Wiley, Hoboken (2010)

Publisher's Note Springer Nature remains neutral with regard to jurisdictional claims in published maps and institutional affiliations 